\title{
A resting pool of vesicles is responsible for spontaneous vesicle fusion at the synapse
}

\author{
Naila Ben Fredj and Juan Burrone \\ MRC Centre for Developmental Neurobiology, King's College London, New Hunt'sHouse, $4^{\text {th }}$ \\ Floor, Guy's Hospital Campus, London, SE1 1UL U.K.
}

\begin{abstract}
Synapses relay information through the release of neurotransmitters stored in presynaptic vesicles. The identity, kinetics and location of vesicle pools mobilized by neuronal activity have been studied using a variety of techniques. Here, we describe a novel genetically-encoded probe, biosyn, which consists of a biotinylated VAMP-2 expressed at presynaptic terminals. We exploit the high affinity interaction between streptavidin and biotin to label biosyn with fluorescent streptavidin during vesicle fusion. This approach allows tagging of vesicles sequentially, to visualize and establish the identity of presynaptic pools. Using this technique we were able to distinguish between two different pools of vesicles in rat hippocampal neurons: one that is released in response to presynaptic activity and another, distinct vesicle pool that spontaneously fuses with the plasma membrane. We further established that the spontaneous vesicles belong to a 'resting pool' that is normally not mobilized by neuronal activity and whose function is mostly unknown.
\end{abstract}

\section{Introduction}

The transfer of information at a synapse requires release of neurotransmitter from a presynaptic terminal and the subsequent activation of postsynaptic receptors. All synapses show both evoked (calcium-dependent) and spontaneous (calcium-independent) forms of neurotransmitter release 1. Evoked release is tightly coupled to the action potential and generates a postsynaptic response with sub-millisecond delay 2. At rest, in the absence of any activity, the machinery responsible for the fusion of neurotransmitter-filled vesicles at a synapse has a very low release probability, but is not zero. As a result, spontaneous fusion events occur stochastically, but at a very low rate, estimated to be around 1-2 vesicle per minute 3,4 . The consensus view is that membrane depolarization (by action potentials or otherwise) increases the probability of vesicle fusion allowing the synapse to switch from a negligible rate of spontaneous release to a rapid synchronized form of release. Recently, a debate has ensued regarding the origin of each mode of release 5,6. Do vesicles that release neurotransmitter spontaneously draw from the same pool as those released in response to neuronal activity? Related to this question, is spontaneous (or constitutive) release an important feature of synaptic transmission? These basic, yet unresolved questions are crucial to understanding information transfer at the synapse and probe some of the basic assumptions of quantal theory 1 . To advance our knowledge in this area it is important to first establish the identity of presynaptic vesicle pools and understand how they are mobilized within a terminal. 
Presynaptic neurotransmitter-filled vesicles are organized into distinct pools, with different kinetics of release and subcellular localization 7. In general, vesicles are thought to be arranged into three main pools: a rapidly releasable pool (RRP), a reserve pool and a resting pool. The sizes of each pool, their location within the synapse, as well as the modes in which they are mobilized are still a matter of debate 7. It is generally thought that the RRP constitutes those vesicles closest to the plasma membrane, docked at the active zone and ready for immediate release 8 . The reserve pool serves to refill the empty release sites at the RRP and acts as a source of vesicles, which the bouton can draw on to continue releasing neurotransmitter after the RRP has been used up. These two pools are collectively known as the recycling pool and are mobilized by membrane depolarization in a calcium-dependent manner. Finally, there is a group of vesicles known as the resting pool, which are refractory to release in response to electrical activity 3,9. The function of this pool of vesicles has remained largely unknown 10 .

Spontaneous events, known as miniature postsynaptic events or minis, are postsynaptic measures that reflect the release of neurotransmitter released from a single vesicle (or quanta) and were used to formulate the quantal theory of neurotransmitter release 1. From a presynaptic perspective, dyes that label membranes, such as FM1-43, have shown that spontaneous vesicle exo- and endocytosis also occur at the synapse and concluded that these events correspond to the postsynaptic mini 3,11. One widely accepted assumption is that vesicles undergoing both spontaneous and activity-dependent fusion use the same set of vesicles and the same fusion machinery. More recent studies have proposed that these two modes of release may draw from different pools 5,12. To date, conflicting reports have found evidence both for and against two separate pools of vesicles, leaving the question unresolved 6,11. Here, we develop and implement a novel technique to label vesicles in live neurons. Using this method, we show the existence of two independent pools of vesicles: one that fuses spontaneously with the plasma membrane and another that can only be mobilized by neuronal activity. In addition, we used the presynaptic probe synaptophysinpHluorin (sypHy) to establish the identity of the spontaneous vesicle pool. We find that spontaneously-released vesicles are mobilized from a 'resting pool' that was originally described as an activity-independent set of vesicles that do not participate in vesicle cycling. Our data not only provides further evidence for the existence of a functionally heterogeneous population of vesicles in presynaptic terminals, but also establishes a role for the previously ill-defined resting pool.

\section{Results}

\section{Biosyn: a new tool for labelling presynaptic vesicle proteins}

We have developed a technique for labelling presynaptic vesicle proteins by combining in vivo biotinylation of transmembrane proteins together with irreversible tagging with fluorescent streptavidin. Our approach is an adaptation of a previously reported tool used to label surface antibodies and other transmembrane proteins 13, including postsynaptic receptors 14 (Fig. 1a). We generated a fusion protein between the presynaptic vesicle protein, VAMP2, and a biotin acceptor peptide (BAP) that is specifically recognised by the biotin ligase enzyme BirA. The BAP domain was fused to the luminal end (C-terminus) of VAMP2 that is topologically equivalent to the extracellular domain. We used an engineered version of the BirA enzyme that localizes to the lumen of the Endoplasmic Reticulum through a signal that translocates proteins to the secretory pathway 13. Co-expression of VAMP-2-BAP and BirA results in a luminal biotinylated VAMP-2, which we termed 'biosyn'. Fluorescently tagged streptavidin can subsequently be used to irreversibly bind biosyn and label newly exocytosed vesicles fluorescently at presynaptic terminals (Fig. 1a). 


\section{Biosyn labels vesicles released by presynaptic activity}

We first set out to establish if we could label biosyn with a fluorescent streptavidin in live presynaptic terminals (Fig. 1b). Neurons expressing Vamp2-BAP, BirA and a presynaptic EGFP-based reporter synaptophysin-pHluorin (sypHy; green puncta in Fig. 1c)15 were incubated in normal HBS containing streptavidin-alexa647 (strep647), to label all the biosyn on the plasma membrane (shown as blue puncta in Fig. 1c). After washing away the strep647, neurons were stimulated with a high potassium solution (see methods) containing streptavidin-alexa555 (strep555) to label all newly exocytosed vesicles from the recycling pool (red puncta in Fig. 1c). The resulting puncta correspond to clusters of vesicles released at presynaptic terminals, which co-localize with the presynaptic marker sypHy (green). The specificity of this technique was further established by performing experiments under conditions that should not result in vesicle cycling, such as depolarization in the absence of calcium, or in the presence of cadmium (a potent calcium channel blocker). Very weak staining was observed under these conditions (staining was not completely absent due to some spontaneous vesicle fusion events, described below), indicating the technique can specifically tag activity-dependent vesicle fusion events (Fig. 1d). We have also established that neurons expressing only BirA or only VAMP2-BAP, showed no staining with fluorescent streptavidin after stimulation with high potassium (Supplementary Fig. 1). Finally, only axons expressing biosyn were labelled with fluorescent streptavidin; all neighbouring untransfected axons showed no fluorescence staining, ruling out any nonspecific binding by streptavidin. It is known that VAMP-2 present on the surface mixes with the vesicular VAMP-2 after exocytosis. In agreement with this, we found that the size of the recycling pool positively correlated with the surface fraction of VAMP-2 at rest (Fig. 1e; correlation coefficient $\mathrm{R}=0.55, \mathrm{p}<0.05$ ).

We next established whether we could tag vesicles released in response to action potentials (Fig. 2). Neurons expressing both sypHy and biosyn were stimulated strongly (900 APs at $20 \mathrm{~Hz}$ ) to mobilize the entire recycling pool of vesicles (Fig. 2a). SypHy is a sensitive reporter of presynaptic vesicle cycling consisting of a fusion protein between synaptopHysin and a $\mathrm{pH}$ sensitive EGFP, pHluorin, which is quenched inside the acidic lumen of a vesicle 15,16 . Upon vesicle fusion the pHluorin molecule increases its fluorescence as it contacts the basic extracellular $\mathrm{pH}$. As a result, this probe provides a sensitive means of assessing the amount of exocytosis that occurs during a stimulation protocol. We used the alkaline trapping method which exploits the properties of bafilomycin (a specific blocker of the vesicular proton pump) to abolish the reacidification of vesicles after endocytosis resulting in cumulative increases in sypHy fluorescence signals that directly correspond to the number of vesicles exocytosed 17. We measured sypHy fluorescence (green channel) during the staining stimulus whilst strep594 was present in the extracellular medium (Fig. 2b,c). We found a linear correlation between the change in fluorescence measured with sypHy $(\Delta \mathrm{F}$ amplitude) and the intensity of strep594 fluorescence at individual synapses (Fig. 2d,e; correlation coefficient $\mathrm{R}=0.81, \mathrm{p}<0.05$ ). Our results clearly show that biosyn faithfully reports vesicle fusion events and can be used to establish the size of vesicle pools at presynaptic terminals. Finally, it was important to establish whether tagging biosyn with streptavidin had any effect on subsequent vesicle exo/endocytosis. Two lines of evidence suggest that labelled synapses behave normally: 1- synapses where the entire recycling pool was stained with streps488 could then be restained with FM4-64 (Supplementary Fig. 2) and 2- neurons co-transfected with sypHy and biosyn showed similar sypHy responses before and after strep594 staining (Supplementary Fig. 3). Together, our data show that biosyn is a reliable probe for labelling exocytosed vesicles in presynaptic terminals. 


\section{Biosyn labels vesicles that fuse spontaneously}

Synapses also undergo spontaneous fusion events, which are independent of neuronal activity. Imaging these events has proven a difficult task with the available tools and has led to important discrepancies in the interpretation of vesicle pool identity. We performed experiments to test whether biosyn could detect these spontaneous events reliably and with sufficient sensitivity. After quenching the surface biotin with strep647 we incubated neurons expressing biosyn with strep555 for 15 minutes at $37^{\circ} \mathrm{C}$, in the presence of tetrodotoxin (TTX) to silence $\mathrm{Na}^{+}$-dependent action potentials and in the absence of extracellular calcium to avoid any activity-dependent release (Fig. 3a). We found that strep555 puncta clearly colocalized with putative synapses identified with the synaptic marker sypHy (Fig. 3b). In addition, the surface strep647 intensity was highly correlated with the intensity of strep555, suggesting that the biosyn surface fraction readily mixes with the biosyn present in vesicles released spontaneously (Fig. 3c; correlation coefficient $R=0.77$, $p<0.05$ ). Indeed, experiments where pulses of streptavidin are delivered to label the surface fraction of biosyn before and after spontaneous fusion have uncovered substantial mixing at the plasma membrane (Ben Fredj and Burrone, unpublished observations). More importantly, we measured a time-course for spontaneous vesicle release (Fig. 3d). Our data shows that there is a finite pool of vesicles that saturates with a time constant of around 6 minutes. As expected, this form of release was temperature dependent, so that at room temperature, after 15 min of spontaneous fusion, only $55 \%$ of the pool was labelled (open circle in Fig. 3d). Our results agree with previous estimates of spontaneous release in hippocampal neurons, showing that most of the spontaneous pool is released within 10-15 min 5,6. However, any rapid transient fusion events 18 may hamper streptavidin binding to biosyn molecules in a vesicle. In such a case our measured time-constant would represent an overestimate of the actual value, although it would have no effect on the total size of the pool.

\section{Spontaneous and evoked vesicle fusion use two different pools of vesicles}

Surprisingly, the size of the spontaneously released pool of vesicles was roughly half the size of the evoked pool (spontaneous pool: $0.46 \pm 0.05, \mathrm{n}=21$ neurons; data normalized to evoked: $1 \pm 0.50, \mathrm{n}=64$ neurons). If both forms of release used the same set of vesicles, we would expect the size of both pools to be equal. This discrepancy can be explained in one of two ways: 1 - only a subset of those vesicles released in response to activity is also competent for spontaneous fusion; 2- each mode of release uses separate pools of vesicles. To tease apart these two possibilities we exploited an important property of biosyn: vesicles that undergo fusion at a presynaptic terminal can be tagged separately and sequentially with different fluorescent markers. In this way, by saturating all the biosyn binding sites of vesicles involved in one mode of release we could then test if the number of vesicles mobilized by the other mode of release was affected.

Synapses were first stimulated with a saturating stimulus of two 90 second depolarizations in the presence of strep488, which strongly labelled the entire recycling pool (Fig. 4a). A further depolarizing stimulus in strep594 resulted in no further labelling (or very small amounts of labelling), as all biosyn binding sites were occupied by strep488, confirming that our depolarizing stimulus mobilized all possible vesicles (Fig. 4a images). On the other hand, after labelling the recycling pool with strep488, a further 15 minute exposure to strep594 in conditions which would only allow spontaneous fusion events resulted in a significant amount of labelling (Fig. 4b; importantly, no evoked vesicles became available for release during the period of spontaneous labelling as shown in Supplementary Fig. 4). The cumulative intensity distribution for spontaneous labelling was very similar when spontaneous release was assayed before or after the evoked pool was released (Fig.4c; solid and dotted line, respectively), suggesting that the depletion of the entire recycling pool of vesicles had no effect on the total size of the spontaneous pool. However, when spontaneous 
labelling was assayed after the spontaneous pool had been previously mobilized, we found a strong reduction in biosyn staining (dashed line), as expected for labelling times close to saturation. Similarly, the cumulative intensity distribution for evoked release was not affected by spontaneous release (Fig.4d; solid and dotted line), but was strongly decreased after a saturating stimulus released the whole recycling pool (dashed line). Central to our choice of stimulation was the need to label vesicle pools with high specificity, without any crosstalk. To achieve this, we exploited the temperature-dependence of spontaneous release (see Fig. 3d) by performing all our high $\mathrm{K}^{+}$stimulations at room temperature, to minimize spontaneous events. In this way we were able to isolate each vesicle pool with minimal contamination. Figure 5a shows the average data compiled from three independent experiments (comprising between 21-65 cells per condition and on average 20 synapses per cell). Here, the data was pooled from experiments using different fluorophores and each condition was normalized to the size of the recycling pool, i.e.: the intensity of labelled biosyn after two consecutive bouts of depolarization. The most obvious feature from our data is that the intensity of spontaneous release was independent of the order in which it was labelled (all spontaneous measurements showed no significance difference between them, $\mathrm{p}>0.05$, one-way ANOVA). This is expected if spontaneous and evoked vesicles are distinct. In agreement with this, the intensity of evoked release was also unchanged whether biosyn was labelled before or after spontaneous labelling (no significant difference, $p>0.05$, oneway ANOVA). Together, our data shows that there are two independent modes of vesicle release at the presynaptic terminal of hippocampal neurons and that each uses a separate set of vesicles.

It has previously been proposed that the amount of spontaneous release at a bouton correlates well with release probability 3 , or a measure of evoked release 11 . We also found a high degree of correlation between the size of the spontaneous and evoked pool of vesicles, measured at the same synapse (Fig. 5b; correlation coefficient $R=0.57, p<0.05$ ). These findings suggest that the properties of spontaneous release may accurately reflect those of evoked release. However, we also note that there are a group of synapses that show a bias towards spontaneous labelling, with low amounts of evoked labelling (Fig. 5b, points on the top left hand side of the graph). We are unsure what these synapses represent, although preliminary data suggests they may constitute newly formed immature synaptic contacts (unpublished observations). More experimental data is needed to establish their identity further.

\section{Identity of the spontaneous pool}

If the recycling pool of vesicles cannot account for spontaneous release, then where do these vesicles come from? At presynaptic terminals, there is a pool of vesicles that cannot be mobilized by neuronal activity and whose function is largely unknown. In general, it is referred to as the 'resting pool' of vesicles 10 and provides a possible candidate for spontaneous release. Previous studies have used pHluorin-based probes together with the alkaline trap method to establish the size of different synaptic vesicle pools, including the resting pool 17,19. Fig. 6A shows how we applied this method on synapses expressing sypHy to measure the relative sizes of the recycling and the resting pools. Axons were stimulated with a saturating stimulus (900 APs at $20 \mathrm{~Hz}$ ), in the presence of the vesicular proton pump antagonist bafilomycin, to mobilize the entire recycling pool. Note how the stimulus results in an increase in fluorescence that does not return to baseline, as any reacidification after endocytosis is inhibited by bafilomycin (Fig. 6a,b). However, there are a number of vesicles that cannot be released during the stimulus and are said to belong to the resting pool. These vesicles may be uncovered by using ammonia (see methods) to homogenize the $\mathrm{pH}$ across all membranes 19 and therefore unquench any vesicles that have not undergone recycling. In this example, ammonia caused a 1.5 fold increase in 
fluorescence, indicating that the resting pool of vesicles was about half the size of the recycling pool. On average, we found that the resting pool contributed an extra $\sim 60-70 \%$ of vesicles, which agrees with previous measurements 19.

To test whether spontaneous fusion drew vesicles from the resting pool we performed a similar experiment to that described in Fig. 6a, only this time, after mobilizing the entire recycling pool, synapses were left to undergo spontaneous release in the presence of bafilomycin. Any increase in fluorescence would represent the spontaneous fusion of vesicles that do not belong to the recycling pool and must therefore belong to the resting pool. An example is shown in Fig. 6b-c. After the stimulus, TTX was added to the medium and synapses were left unstimulated for $15 \mathrm{~min}$. at room temperature. Our previous experiments looking at the time-course of spontaneous fusion had already established that, at this temperature, $55 \%$ of the spontaneous pool would be mobilized (see Fig. 3d). In agreement with this, we found an increase in fluorescence that represented about $60 \%$ of the resting pool. The total size of the resting pool was established by ammonium unquenching, which in this example cell was quite large (as large as the recycling pool). The pooled data from all our cells is shown in Fig.7a, where values have been normalized to the initial size of the recycling pool. On average, synapses had a resting pool size which was just over half the size of the recycling pool (control synapses: $59 \pm 0.12 \%$; synapses following spontaneous release: $71 \pm 0.26 \%$; not statistically different). Importantly, spontaneous fusion at room temperature reduced the number of vesicles that were subsequently unquenched by ammonia by two thirds (red trace in Fig.7a), indicating that this mode of release was using vesicles from the resting pool. Our data using sypHy confirms that there are two independent pools of vesicles with distinct modes of release and that spontaneous fusion draws vesicles from the resting pool.

Finally, we found that the total size of the recycling pool correlated with the amount of spontaneous release that occurred during the $15 \mathrm{~min}$ period at room temperature (Fig. 7b). This agrees with our findings using biosyn (Fig. 5b), but do not follow recent findings using a similar probe 20 . However, previous studies have also shown a clear correlation between the size of the recycling pool and the size of the spontaneous vesicle pool 5 or between a measure of evoked release and spontaneous vesicle fusion 3,11. Our data, using two different probes, confirms a significant correlation exists between the sizes of the two pools of vesicles.

\section{Discussion}

We have developed a new genetically-encoded probe, biosyn, to irreversibly label vesicular proteins and have used it to study vesicle cycling in presynaptic terminals. We show that biosyn can be used to report vesicle fusion events that occur in response to neuronal activity, as well as the less frequent spontaneous fusion events that occur stochastically at presynaptic boutons. Importantly, this technique allows for labelling of subsets of vesicles sequentially and with different fluorescent markers. Exploiting this property we were able to distinguish two pools of vesicles with very different properties. One set of vesicles could be mobilized by neuronal activity in a calcium-dependent manner and corresponds to the well characterized recycling pool. We found that there also exists another independent set of vesicles that fuse with plasma membrane spontaneously in a calcium-independent manner. These vesicles do not form part of the recycling pool, but instead belong to a resting pool of vesicles, whose role was previously unknown. Critical evidence to support our conclusions comes from the double pulse experiments (shown in Fig. 4 and quantified in Fig. 5), where each vesicle pool is labelled sequentially with two fluorescent streptavidins. We found substantial labelling of spontaneous fusion events at synapses where the recycling pool had been previously tagged. Further still, the size of the spontaneous pool was the same when 
measured before or after the recycling pool had been saturated. Our data argues that there exists a pool of vesicles that recycles constantly at rest and that is refractory to neuronal activity.

\section{A constitutively recycling resting pool of vesicles}

Vesicles are organized into distinct pools. Broadly speaking, there are three pools of vesicles in presynaptic terminals: a rapidly releasable pool, a reserve pool and a resting pool 7. The first two constitute the recycling pool and are those vesicles that are mobilized in response to neuronal activity. The role of the resting pool has been less well understood as its defining characteristic is that it is refractory to neuronal activity 10. Experimentally, the resting pool was originally defined as those vesicles that could not be stained with FM dyes during stimulation. Photoconversion studies revealed that even after strong stimuli only a subset of vesicle within the synapse had taken up the dye 9 . Parallel studies showed a mismatch between the number of vesicles counted after staining the recycling pool with FM dyes and the much larger number of synapses observed in EM reconstructions of synaptic boutons 3,21 . More recently, measurements of vesicle pool sizes using spH also found a resting pool with roughly similar characteristics 19,22 . The size of this pool was found to vary greatly along an axon and did not show any obvious correlation with the size of the recycling pool 22. Similar pools were also reported in the drosophila neuromuscular junction, using spH 23 and FM dyes 24, although it is thought that this pool can be mobilized when vesicle endocytosis is blocked 23. We performed similar experiments with the genetically-encoded reporter of vesicle cycling, sypHy, and found that spontaneous release drew vesicles from precisely the resting pool. Our results not only provides evidence for the existence of two pools of vesicles with different release modes, but also sheds light on the function of the resting pool within the synapse.

\section{A possible role for spontaneous fusion events}

An intriguing possibility raised by our experiments is that spontaneous fusion may in fact represent spontaneous neurotransmitter release at the synapse and therefore correspond to miniature events. Previous findings have shown evidence both for and against separate pools of vesicles involved in evoked and spontaneous neurotransmitter release 6,25. A recent study in dissociated hippocampal neurons used FM dyes together with electrophysiology to describe the existence of a pool of vesicles which spontaneously fuses with the plasma membrane, but which is only reluctantly mobilized by neuronal activity 5 . They hypothesise that vesicles released spontaneously hardly mix with the reserve pool, but instead cycle constitutively and somewhat independently of neuronal activity. More recently, a study in GABAergic neurons suggested spontaneous and evoked pools of vesicles coexist at the synapse and could be modulated independently by kainate 12 . However, these controversial findings have been disputed 6 , in agreement with a previous study that found spontaneously released vesicles, in a subset of synapses, could also be mobilized by neuronal activity 11 . It is unclear why these discrepancies have arisen since all studies used similar systems as well as similar techniques based mainly on FM dye destaining kinetics. However, the method of FM staining and destaining has some drawbacks that could complicate data analysis and interpretation. Long-term exposure of neuronal cultures to FM dyes, needed for staining spontaneous release, can result in increased background signal and non-specific labelling. Our technique overcomes this drawback by using fluorescent streptavidin as a marker, which is an inert protein that can easily be washed off after long periods of exposure. Also, the general approach to analysing FM dye destaining curves is to subtract the background fluorescence after the stimulus and normalize the trace to the initial fluorescence before stimulation. As a result, any vesicles that could not be released by the stimulus will be considered background and be subtracted away 6 . This leaves open the possibility that after staining for spontaneous fusion, a large fraction of vesicles may be left behind but dismissed 
as background. Our approach measures exocytosis directly and does not depend on subsequent destaining to extrapolate the identity of a vesicle.

\section{Molecular identity of vesicle pools}

More indirect evidence for the existence of two pools with distinct modes of release comes from a number of studies showing differences in the properties and molecular identity of the two release modes. Knock-out mice lacking the calcium sensor for vesicle fusion, synaptotagmin I, show a strong reduction in evoked neurotransmitter release, but no change in mini frequency and an important increase in asynchronous release 26,27. This trend is present to some degree in transgenic mice that lack the SNARE protein SNAP-25. Knockout mice for this gene show a marked reduction in evoked release, but vesicles were still competent to fuse spontaneously, although at a lower frequency 28. More surprisingly, mice lacking another SNARE protein, VAMP-2 (synaptobrevin-2), dramatically reduce both spontaneous and evoked release, but also results in mixing between the two pools of vesicles suggesting a role for VAMP-2 in the segregation of each mode of release 5. In Drosophila, mutants for either neuronal synaptobrevin (n-syb)29, syntaxin 30 or cystein string protein (csp) 31, selectively abolish nerve-evoked neurotransmitter release, leaving spontaneous release relatively unperturbed. An alternative phenotype observed in some conditions is an increase in spontaneous release. Overexpression of syt12 (a low-affinity calcium sensor) in dissociated neurons 32 or loss of complexin I drosophila 33 results in an increase in mEPSC frequency, with either no or only small changes in EPSC amplitude, respectively. However, a loss of complexin 1 and 2 in rat hippocampal neurons shows a strong increase in spontaneous mini frequency at the expense of evoked release 34 . Together, these findings suggest that spontaneous release has a different molecular requirement to evoked release. Such indirect evidence adds strength to idea that each mode of release is independent. Nevertheless, the precise molecular composition of each set of vesicles still remains to be established.

One unknown aspect of our findings is the subcellular distribution and fusion sites for each mode of release. For example, it is possible that the spontaneous fusion events described here occur at sites removed from the active zone, yet still within the boundaries of the bouton. At ribbon synapses of retinal bipolar neurons, depolarization evoked release occurred at the active zone, whereas spontaneous fusion was predominant in neighbouring areas, at a distance from the ribbon 35 . Such behaviour may result in spontaneous neurotransmitter release sampling a distinct population of postsynaptic receptors from those used by evoked release. In line with this, a recent study has proposed that minis activate a distinct, yet partially overlapping set of NMDA receptors, than those activated by evoked release 20. This thought provoking finding suggests that minis and evoked neurotransmitter release may occur in different locations at the synapse and exert their influence over distinct patches of the postsynaptic spine. Higher resolution imaging experiments may distinguish between fusion events in different regions of the presynaptic terminal 36 .

\section{Methods}

\section{Hippocampal cultures and transfection}

Primary hippocampal cultures were prepared from embryonic day 18 Sprague-Dawley rats. Hippocampi were dissociated using trypsin $\left(5 \mathrm{mg} / \mathrm{ml}\right.$ for $15 \mathrm{~min}$ at $37^{\circ} \mathrm{C}$, Worthington, UK), triturated and plated onto coverslips coated with poly-D-lysine $(50 \mu \mathrm{g} / \mathrm{ml})$ and laminin $(20 \mu \mathrm{g} / \mathrm{ml})$. Neurons were incubated at $37^{\circ} \mathrm{C}$ under $5 \% \mathrm{CO} 2$ in Neurobasal-based culture media supplemented with B27, glutamax (Gibco) and pen/strep. After 6-7 days in culture, neurons were transfected using Lipofectamine 2000 (Invitrogen). Experiments were done at around 14 days in vitro, when synapses are deemed mature. 


\section{Constructs and plasmids}

Fusion proteins were made between VAMP2 and the biotin acceptor peptide sequence with a small linker sequence separating them. The BAP sequence was obtained by annealing the following oligonucleotides: $5^{\prime}$ - CC GGT GGC CTG AAC GAT ATT TTC GAA GCT CAG AAA ATC GAA TGG CAC GAA GGC GGC TCT TAA T - $3^{\prime}$ and $5^{\prime}$ - CT AGA TTA AGA GCC TTC GTG CCA TTC GAT TTT CTG AGC TTC GAA AAT ATC GTT CAG GCC A $-3^{\prime}$. This results in a BAP sequence (GLNDIFEAQKIEWHE) with two sticky end tails that we used to ligate it into the C-terminal end of a VAMP2-linker construct using AgeI/XbaI. The VAMP2-linker construct was originally obtained from Dr. Missenbock as part of a VAMP2-linker-pHluorin construct cloned into a pCI plasmid (Promega). We removed the pHluorin and replaced it with the BAP domain, resulting in a VAMP2-linker-BAP fusion. We refer to this construct as VAMP2-BAP.

The BirA enzyme (GenBank: P06709) expression plasmid (pCDNA3) was kindly donated by Dr. O. Burrone (Predonzani et al., 2008). It consists of the E. Coli biotin ligase enzyme, BirA, with a secretion sequence added to its N-terminus, resulting in a pCDNA3-sec-BirA plasmid.

\section{Labelling and imaging vesicles with Biosyn}

Primary hippocampal neurons were co-transfected with VAMP2-BAP and BirA plasmids at 6-7 div. Immediately following transfection, $100 \mu \mathrm{M}$ of biotin was added to the medium. Experiments were carried out one week later to ensure that synapses had reached maturity. Experiments were performed in Hepes buffer saline (HBS) (in mM): $139 \mathrm{NaCl}, 2.5 \mathrm{KCl}, 10$ Hepes, 10 D-glucose, $2 \mathrm{CaCl}_{2}, 1.3 \mathrm{MgCl}_{2}$ (pH 7.3 and $290 \mathrm{mOsmol}$ ). Neurons were initially washed in HBS to remove excess biotin and incubated for $30 \mathrm{~s}$ in streptavidin-Alexa 647 (1:250, Invitrogen) to label all biotinylated VAMP2 present at the surface of the plasma membrane. To label the recycling pool of synaptic vesicles, neurons were washed a further three times with HBS to remove excess strep-647 and then depolarized twice for 90 seconds by incubating in a high $\mathrm{K}^{+}$solution containing (in $\mathrm{mM}$ ): $78.5 \mathrm{NaCl}, 60 \mathrm{KCl}, 10 \mathrm{D}$-glucose, 10 HEPES, $2 \mathrm{CaCl}_{2}, 1.3 \mathrm{MgCl}_{2}$, streptavidin-Alexa 488 (1:250), 0.001 TTX, 0.025 APV and $0.02 \mathrm{CNQX}$. Each depolarization was separated by $5 \mathrm{~min}$. After this treatment, the last depolarization was followed by a $5 \mathrm{~min}$ rest before the fixation. To label vesicles cycling spontaneously, neurons were washed 3 times with calcium-free HBS and incubated for 15 minutes at $37^{\circ} \mathrm{C}$ in the presence of streptavidin-Alexa 555, $1 \mu \mathrm{M}$ TTX, $25 \mu \mathrm{M}$ APV and 20 $\mu \mathrm{M} C \mathrm{CNQX}$. Cells were then rinsed in HBS and fixed. The fixation procedure consisted of a 30 minute incubation with $4 \% \mathrm{PFA} / 0.33 \mathrm{M}$ sucrose. Images were obtained using either a Zeiss LSM 510 confocal, a FV1000 Olympus microscope or on an inverted microscope Olympus IX71 using a CCD camera and Slidebook software (Intelligent Imaging Innovations. Santa Monica). Synapses stained with alexa 488, alexa 555 and alexa 647, where imaged on the confocal microscopes with the proper filters. The experiments on the wide field imaging setup (Olympus IX71) used cells stained with alexa 488 and alexa 594 with following filters: excitation filters: $470+/-15 \mathrm{~nm}$ band pass and $565+/-22 \mathrm{~nm}$ band pass filters; dichroic: $515+/-20 \mathrm{~nm}$ band pass, $590 \mathrm{~nm}$ long pass; emission filter: $510+/-$ $15 \mathrm{~nm}$ band pass and $650+/-36 \mathrm{~nm}$ band pass filters. Excitation and emission filters were alternated using a filter wheel. We made sure there was no significant bleed through between channels and, when using confocal microscopy, images were scanned sequentially with each laser to avoid crosstalk.

\section{SynaptopHysin-pHluorin imaging}

SypHy plasmid was a gift from Dr. L. Lagnado. Experiments were done as previously described (Li et al., 2004). Briefly, coverslips were mounted in a custom-made chamber equipped with a pair of parallel platinum electrodes $\sim 5 \mathrm{~mm}$ apart. Neurons were stimulated 
by delivering a 1ms, $25 \mathrm{~mA}$ current pulses using an SD9 stimulator (Grass Instruments), whose timing was controlled by a TTL signal from the imaging software (Slidebook). Bafilomycin A (Calbiochem) was used at a final concentration of $1 \mu \mathrm{M}$. Images were obtained using an inverted microscope, Olympus IX71 with a CCD camera (coolsnap HQ) controlled by Slidebook software (Intelligent Imaging Innovations, Santa Monica). The light source was a xenon-arc lamp (Lambda LS, Sutter), where light exposure was regulated by a rapid shutter (Sutter smartShutter) controlled by a Sutter Instruments lambda 10-3 controller, fitted with a $470 \pm 20 \mathrm{~nm}$ bandpass excitation filter (Chroma Tech. Corp.) and suitable neutral density filters. Only neurons that showed more than a $20 \%$ increase in fluorescence on average after treatment with ammonia were used. A small number of neurons ( 6 out of 21 neurons) did not show an increase in fluorescence and these cells were excluded from the analysis.

\section{Data Analysis}

Images were exported as TIFF files and were analyzed using custom-written routines in MATLAB (MathWorks Inc., Natick,MA) as described previously (Li et al., 2004). Briefly, square regions of interests (ROI's; of $8 \times 8$ pixels in width) were drawn around synapses (generally 4-6 pixels in diameter) and the average fluorescence intensity for each synapse was calculated at its best focal point. When analysing data from boutons stained for both spontaneous and evoked fusion events, ROI's were selected in the channel used for labelling evoked events and the same ROI coordinates were then applied to other channels. In this way we made sure to take only synapses that had activity-dependent labelling. Statistical comparisons were made by using either ANOVA or t-tests. Statistical analysis was performed with Prism software. Normality was assessed using d'Agostino and Pearson omnibus normality test. If samples follow a Gaussian distribution, we used the appropriate parametric tests. Where this assumption is not met, we used a non-parametric test. Descriptive statistics are reported as mean \pm SEM.

\section{Supplementary Material}

Refer to Web version on PubMed Central for supplementary material.

\section{Acknowledgments}

We would like to thank O. Burrone for kindly donating the modified BirA expression construct and for helpful advice and discussion on experimental protocols. We would also like to thank I. Thompson and the members of the Thompson and Burrone laboratories for helpful discussions and suggestions. We would finally like to thank V.N. Murthy for his input in the initial stages of this project and L. Lagnado, Q. Ch'ng, M. Grubb and L. Andreae for critical reading of the manuscript. This work was supported by a Medical Research Council project grant and a Wellcome Trust project grant to J.B.

\section{References}

1. Katz B. The release of neural transmitter substances. 1969

2. Sabatini BL, Regehr WG. Timing of synaptic transmission. Annu Rev Physiol. 1999; 61:521-42. [PubMed: 10099700]

3. Murthy VN, Stevens CF. Reversal of synaptic vesicle docking at central synapses. Nat Neurosci. 1999; 2:503-7. [PubMed: 10448213]

4. Geppert M, et al. Synaptotagmin I: a major $\mathrm{Ca} 2+$ sensor for transmitter release at a central synapse. Cell. 1994; 79:717-27. [PubMed: 7954835]

5. Sara Y, Virmani T, Deak F, Liu X, Kavalali ET. An isolated pool of vesicles recycles at rest and drives spontaneous neurotransmission. Neuron. 2005; 45:563-73. [PubMed: 15721242]

6. Groemer TW, Klingauf J. Synaptic vesicles recycling spontaneously and during activity belong to the same vesicle pool. Nat Neurosci. 2007; 10:145-7. [PubMed: 17220885] 
7. Rizzoli SO, Betz WJ. Synaptic vesicle pools. Nat Rev Neurosci. 2005; 6:57-69. [PubMed: 15611727]

8. Schikorski T, Stevens CF. Morphological correlates of functionally defined synaptic vesicle populations. Nat Neurosci. 2001; 4:391-5. [PubMed: 11276229]

9. Harata N, et al. Limited numbers of recycling vesicles in small CNS nerve terminals: implications for neural signaling and vesicular cycling. Trends Neurosci. 2001; 24:637-43. [PubMed: 11672807]

10. Sudhof TC. The synaptic vesicle cycle revisited. Neuron. 2000; 28:317-20. [PubMed: 11144340]

11. Prange O, Murphy TH. Correlation of miniature synaptic activity and evoked release probability in cultures of cortical neurons. J Neurosci. 1999; 19:6427-38. [PubMed: 10414971]

12. Mathew SS, Pozzo-Miller L, Hablitz JJ. Kainate modulates presynaptic GABA release from two vesicle pools. J Neurosci. 2008; 28:725-31. [PubMed: 18199771]

13. Predonzani A, Arnoldi F, Lopez-Requena A, Burrone OR. In vivo site-specific biotinylation of proteins within the secretory pathway using a single vector system. BMC Biotechnol. 2008; 8:41. [PubMed: 18423015]

14. Chen I, Howarth M, Lin W, Ting AY. Site-specific labeling of cell surface proteins with biophysical probes using biotin ligase. Nat Methods. 2005; 2:99-104. [PubMed: 15782206]

15. Granseth B, Odermatt B, Royle SJ, Lagnado L. Clathrin-mediated endocytosis is the dominant mechanism of vesicle retrieval at hippocampal synapses. Neuron. 2006; 51:773-86. [PubMed: 16982422]

16. Miesenbock G, De Angelis DA, Rothman JE. Visualizing secretion and synaptic transmission with pH-sensitive green fluorescent proteins. Nature. 1998; 394:192-5. [PubMed: 9671304]

17. Burrone J, Li Z, Murthy VN. Studying vesicle cycling in presynaptic terminals using the genetically encoded probe synaptopHluorin. Nat Protoc. 2006; 1:2970-8. [PubMed: 17406557]

18. Aravanis AM, Pyle JL, Tsien RW. Single synaptic vesicles fusing transiently and successively without loss of identity. Nature. 2003; 423:643-7. [PubMed: 12789339]

19. Li Z, et al. Synaptic vesicle recycling studied in transgenic mice expressing synaptopHluorin. Proc Natl Acad Sci U S A. 2005; 102:6131-6. [PubMed: 15837917]

20. Atasoy D, et al. Spontaneous and evoked glutamate release activates two populations of NMDA receptors with limited overlap. J Neurosci. 2008; 28:10151-66. [PubMed: 18829973]

21. Schikorski T, Stevens CF. Quantitative ultrastructural analysis of hippocampal excitatory synapses. J Neurosci. 1997; 17:5858-67. [PubMed: 9221783]

22. Fernandez-Alfonso T, Ryan TA. A heterogeneous "resting" pool of synaptic vesicles that is dynamically interchanged across boutons in mammalian CNS synapses. Brain Cell Biol. 2008; 36:87-100. [PubMed: 18941900]

23. Poskanzer KE, Davis GW. Mobilization and fusion of a non-recycling pool of synaptic vesicles under conditions of endocytic blockade. Neuropharmacology. 2004; 47:714-23. [PubMed: 15458843]

24. Delgado R, Maureira C, Oliva C, Kidokoro Y, Labarca P. Size of vesicle pools, rates of mobilization, and recycling at neuromuscular synapses of a Drosophila mutant, shibire. Neuron. 2000; 28:941-53. [PubMed: 11163278]

25. Sara Y, et al. Selective capability of SynCAM and neuroligin for functional synapse assembly. $\mathrm{J}$ Neurosci. 2005; 25:260-70. [PubMed: 15634790]

26. Nishiki T, Augustine GJ. Synaptotagmin I synchronizes transmitter release in mouse hippocampal neurons. J Neurosci. 2004; 24:6127-32. [PubMed: 15240804]

27. Chapman ER. How does synaptotagmin trigger neurotransmitter release? Annu Rev Biochem. 2008; 77:615-41. [PubMed: 18275379]

28. Washbourne P, et al. Genetic ablation of the t-SNARE SNAP-25 distinguishes mechanisms of neuroexocytosis. Nat Neurosci. 2002; 5:19-26. [PubMed: 11753414]

29. Deitcher DL, et al. Distinct requirements for evoked and spontaneous release of neurotransmitter are revealed by mutations in the Drosophila gene neuronal-synaptobrevin. J Neurosci. 1998; 18:2028-39. [PubMed: 9482790] 
30. Schulze KL, Broadie K, Perin MS, Bellen HJ. Genetic and electrophysiological studies of Drosophila syntaxin-1A demonstrate its role in nonneuronal secretion and neurotransmission. Cell. 1995; 80:311-20. [PubMed: 7834751]

31. Umbach JA, et al. Presynaptic dysfunction in Drosophila csp mutants. Neuron. 1994; 13:899-907. [PubMed: 7946336]

32. Maximov A, Shin OH, Liu X, Sudhof TC. Synaptotagmin-12, a synaptic vesicle phosphoprotein that modulates spontaneous neurotransmitter release. J Cell Biol. 2007; 176:113-24. [PubMed: 17190793]

33. Huntwork S, Littleton JT. A complexin fusion clamp regulates spontaneous neurotransmitter release and synaptic growth. Nat Neurosci. 2007; 10:1235-7. [PubMed: 17873870]

34. Maximov A, Tang J, Yang X, Pang ZP, Sudhof TC. Complexin controls the force transfer from SNARE complexes to membranes in fusion. Science. 2009; 323:516-21. [PubMed: 19164751]

35. Zenisek D. Vesicle association and exocytosis at ribbon and extraribbon sites in retinal bipolar cell presynaptic terminals. Proc Natl Acad Sci U S A. 2008; 105:4922-7. [PubMed: 18339810]

36. Willig KI, Rizzoli SO, Westphal V, Jahn R, Hell SW. STED microscopy reveals that synaptotagmin remains clustered after synaptic vesicle exocytosis. Nature. 2006; 440:935-9. [PubMed: 16612384] 


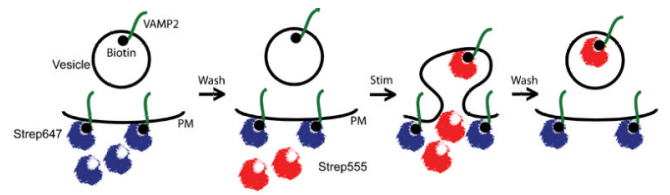

b
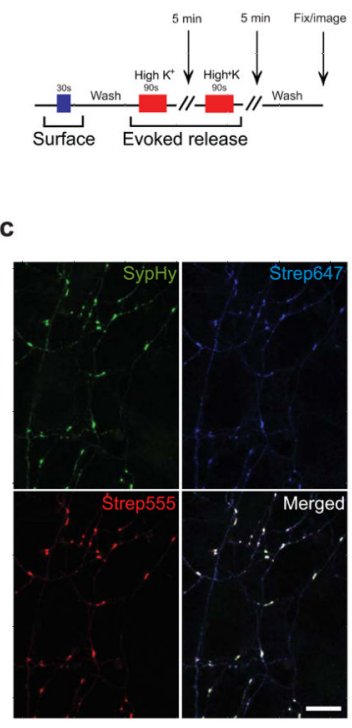

d
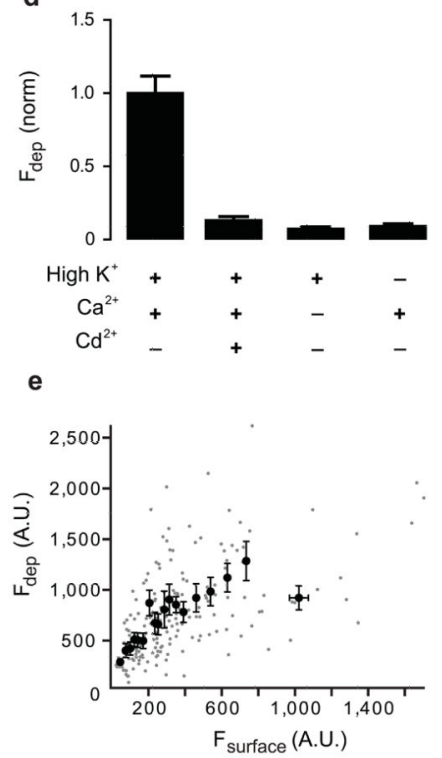

Figure 1. Visualizing vesicles released by membrane depolarization using biosyn (a) Diagram showing labelling of biosyn with streptavidin. Neurons are first pre-treated with streptavidin Alexa647 (strep647, shown in blue) to quench surface VAMP2. Excess streptavidin is removed by washing. The neurons are subsequently stimulated in the presence of streptavidin Alexa555 (strep555, shown in red) to label the vesicles that fuse with the plasma membrane during neurotransmitter release and expose their intraluminal biosyn molecules to the extracellular space. (b) Schematic diagram showing the timeline of the experimental protocol. Surface biosyn was stained with strep647 (blue bar) for 30 sec., washed and then neurons were stimulated twice with a high $\mathrm{K}^{+}$solution in the presence of strep555 (red bar; 5 min interval between depolarizations). After washing, neurons were fixed and imaged. (c) Hippocampal neurons were co-transfected with biosyn and synaptophysin-pHluorin (SypHy). Images show co-localisation of individual synapses expressing SypHy (green), with the recycling pool of vesicle labelled with strep555 (red) and surface labelling with strep647 (blue). Scale bar $=10 \mu \mathrm{m}$. (d) Graph showing a plot of the fluorescence intensity of synapses labelled by depolarization as a function of surface label. Individual synapses are shown in gray and the binned (groups of 10) values in black (n = 445 synapses from 20 cells). (e) Quantification of biosyn staining under different conditions. High $\mathrm{K}^{+}$stimulation specifically labels the activity dependent pool when calcium is present. All conditions were normalized to this value. Staining is abolished when calcium is absent or when cadmium (a calcium channel blocker) is present ( $n>200$ synapses from 10 cells per condition). Values are shown as mean \pm SEM. 
a

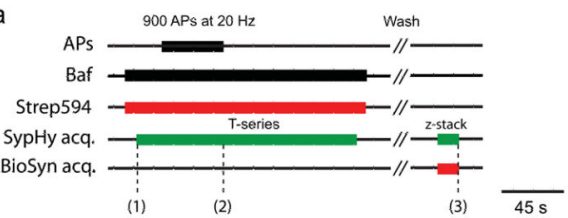

b

d
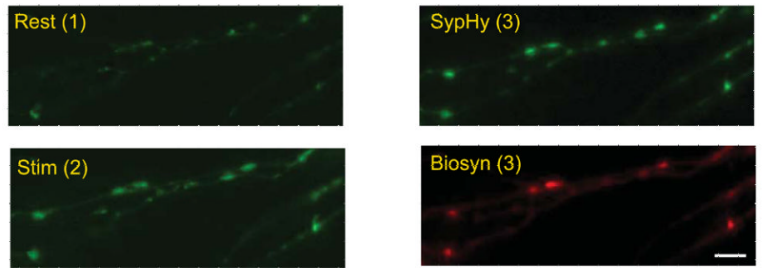

c
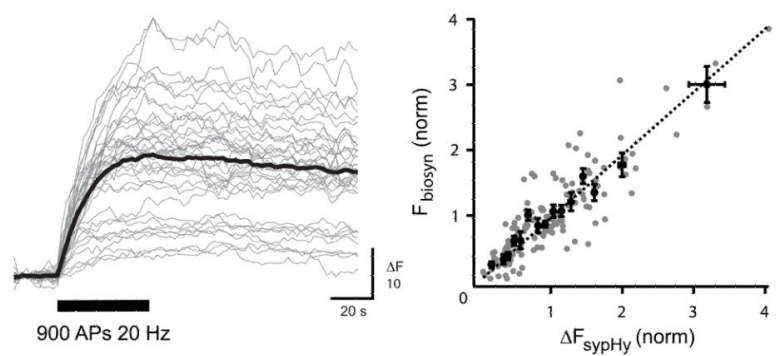

Figure 2. Biosyn used as a reliable tool to assess evoked vesicle fusion

Hippocampal neurons were co-transfected with biosyn and sypHy. (a) Schematic diagram showing the timeline of the experimental protocol: neurons were stimulated with 900 APs at $20 \mathrm{~Hz}$ in the presence of strep555 to label biosyn, while measuring the amount of exocytosis reported by synaptophysin-pHluorin (sypHy) in the green channel. (b) Images show sypHy fluorescence before and immediately after the stimulus. (c) Responses to 900 APs at $20 \mathrm{~Hz}$ (black bar) measured from all synapses analyzed from a single cell (gray) with the average response (black) overlayed. (d) After the stimulus and having washed away the strep555, synapses were found to be strongly labelled. Note the red puncta clearly co-localise with sypHy. Scale bar $=5 \mu \mathrm{m}$. (e) The graph plots the fluorescence intensity of biosyn for individual synapses as a function of the change in fluorescence measured with sypHy $(\mathrm{n}=$ 145 synapses from 6 cells). The black line represents the best linear fit to the data set, constrained to go through the origin. Values are shown as mean \pm SEM. 


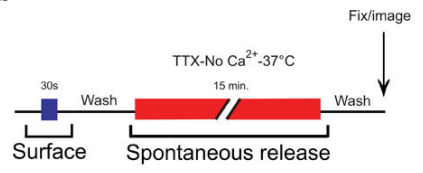

b
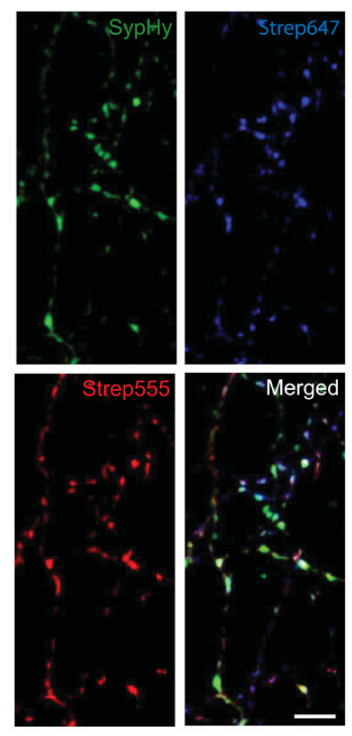

C

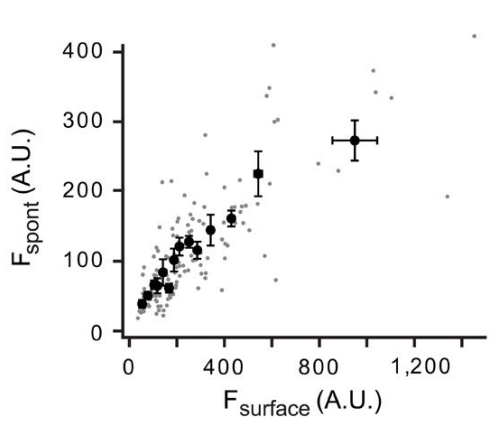

d

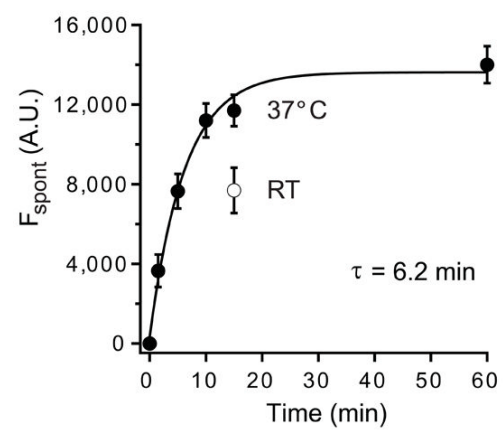

Figure 3. Visualising vesicles released spontaneously with biosyn

(a) Schematic diagram showing the timeline of the experimental protocol. After labelling surface biosyn with strep647 (blue bar) for $30 \mathrm{sec}$., spontaneous vesicle fusion was labelled with strep555 (red bar) in the presence of TTX, nominally zero calcium and incubated at $37^{\circ}$ $\mathrm{C}$ for $15 \mathrm{~min}$. After washing, neurons were fixed and imaged. (b) Hippocampal neurons were co-transfected with biosyn and sypHy. Images show individual synapses expressing sypHy (green), spontaneous vesicle fusion events labelled with strep555 (red) and surface biosyn labelled with strep647 (blue). Scale bar $=5 \mu \mathrm{m}$. (c) Plot of fluorescence intensity for spontaneous versus surface biosyn for individual synapses $(n=545$ synapses from 28 cells). (d) Time course of spontaneous vesicle fusion. Hippocampal neurons were treated with strep555 for different periods of time. The graph shows the fluorescence intensity of spontaneous labelling as a function of time exposed to strep555. The data was fit with the following exponential function: $F=F_{\max }-F_{1} \exp ^{(-t / \tau)}$, where $F$ is the fluorescence of spontaneous release, $F_{\max }$ is the maximum fluorescence, $t$ is time and $\tau$ is the time constant. We find a pool of vesicles is released spontaneously with a time-constant $(\tau)$ of $6.2 \mathrm{~min}$ at $37^{\circ} \mathrm{C}$ (filled circles; $\mathrm{n}=18-25$ cells per condition). The open circle is a single time point at 15 min performed at room temperature. Values are shown as mean \pm SEM. 

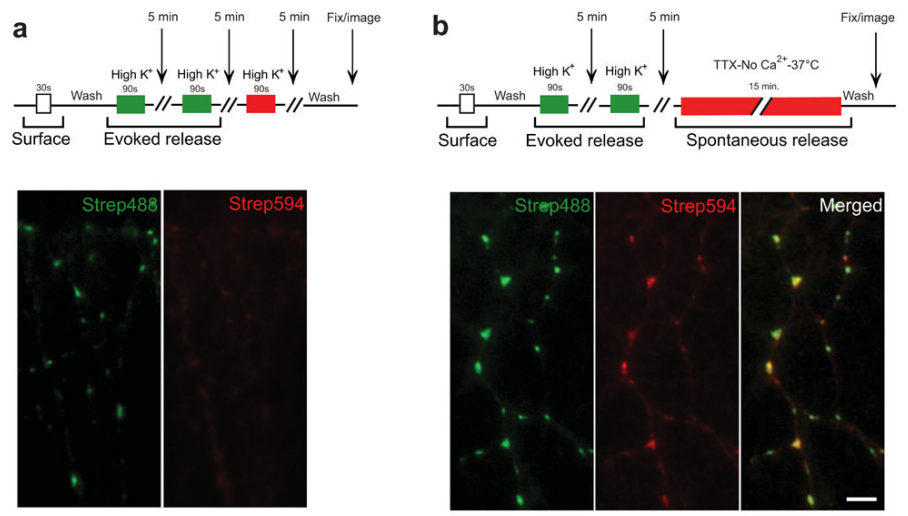

C

Spontaneous

d

Depolarization
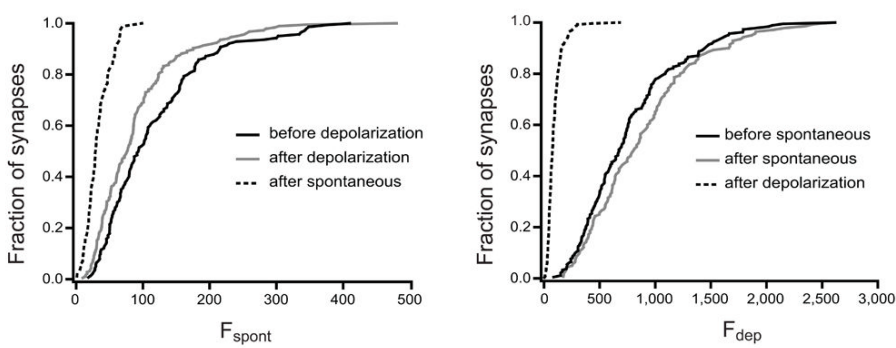

Figure 4. Two distinct pools of vesicle with different release modes: spontaneous and evoked (a) Two consecutive depolarisations with strep488 (green) label the entire recycling pool. A further depolarisation with strep594 (red) results in no further labelling. (b) Labelling the recycling pool (green) followed by spontaneous labelling (red) results in a strong signal in both channels, suggesting that each set of vesicles draws from two distinct pools. Scale bar = $5 \mu \mathrm{m}$. (c) Example from one set of sister cultures showing the cumulative distribution of fluorescence intensity for synapses labelled with strep594 for spontaneous fusion before (grey line, $\mathrm{n}=140$ synapses) and after (solid line, $\mathrm{n}=185$ synapses) depolarization (the median intensity is not significant between the two conditions; one-way ANOVA, $p>0.05$ ). The dashed line shows spontaneous labelling after the entire pool of spontaneous vesicles was labelled with another colour ( $n=85$ synapses; statistically significant when compared to either of the two conditions plotted in the graph; one-way ANOVA, $\mathrm{p}<0.001$ ). Note that the depletion of the recycling (activity-dependent) pool does not affect the size of the spontaneous pool. (d) Cumulative distribution of fluorescence intensity for synapses from the same sister cultures labelled with strep488 using a depolarizing stimulus before (solid line, $\mathrm{n}=185$ synapses) and after (grey line, $\mathrm{n}=140$ synapses) the spontaneous pool is released (the median intensity is not significant between the two conditions; one-way ANOVA, $\mathrm{p}>0.05)$. The dashed line shows evoked labelling after the entire pool of evoked vesicles are labelled with another colour $(n=263$ synapses; statistically significant when compared to either of the two conditions plotted in the graph; one-way ANOVA, $\mathrm{p}<0.001$ ). Note that (c) and (d) use different fluorescent probes (strep594 and strep488, respectively). Fluorescence intensities can only be compared between curves of the same graph. Values are shown as mean \pm SEM. 


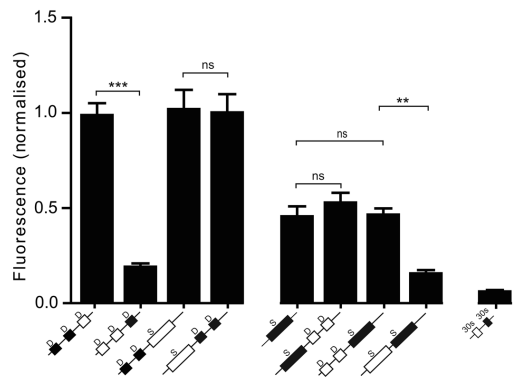

b

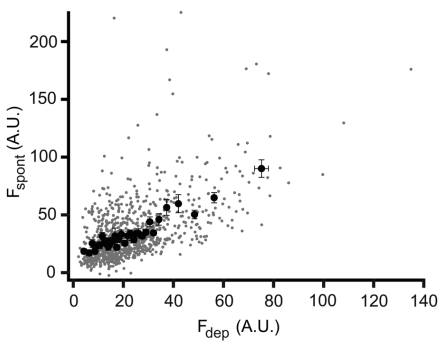

Figure 5. Quantification of fluorescence intensity for different conditions

(a) The graph shows the fluorescence intensity of synapses labelled with a depolarizing stimulus (D) or during spontaneous release (S). Each condition involves several labelling protocols, the sequence of which is shown below each bar. The fluorescence intensity plotted corresponds to that shown as the filled squares/rectangles (black), whereas the empty squares/rectangles are treatments that are not plotted above. The first two bars (from the left) show the intensity of vesicles labelled with depolarizing stimuli, delivered before or after another depolarizing stimulus ( $n=64$ neurons for each bar). The next two bars also represent the intensity of vesicles labelled with depolarizing stimuli, this time before or after spontaneous release $(n=28$ and $n=32$ neurons, respectively). The next set of four bars corresponds to the intensity of vesicles labelled spontaneously. Regardless of when spontaneous release is assessed (on its own, before or after a depolarizing stimulus) the total intensity of spontaneous labelling is very similar $(n=21, n=27$ and $n=32$ neurons, respectively). Note that spontaneous labelling after the spontaneous pool was mobilized is significantly reduced, indicating pool saturation. The final bar is a control to show that surface labelling of biosyn (30 sec exposure to streptavidin) saturates all biosyn binding sites ( $\mathrm{n}=14$ neurons). Statistical analysis was performed using a non-parametric one way a ANOVA test. ns: not significant ( $p>0.05)$. (b) Graph showing the fluorescence intensity of the spontaneous pool as a function of the fluorescence intensity of the recycling pool, for individual synapses (gray dots) and grouped in bins of 30 (black circles; $n=989$ synapses from 26 cells). Note the significant degree of correlation (correlation coefficient $=0.57, \mathrm{p}<$ $0.05)$. Values are shown as mean \pm SEM. 
a

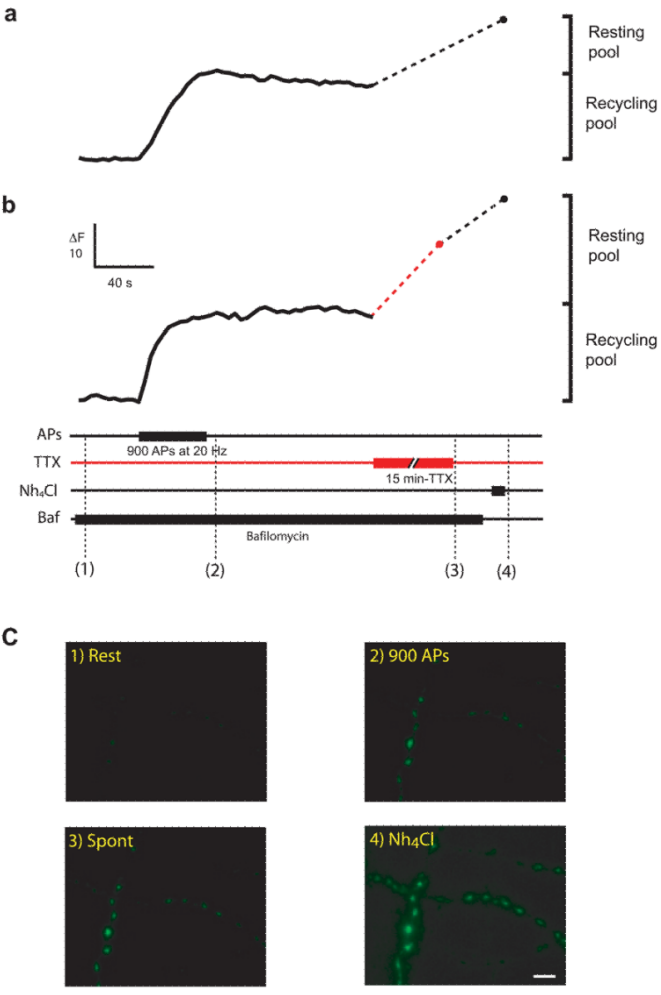

Figure 6. Measuring vesicle pools with sypHy

(a) Changes in fluorescence from a single neuron in response to $900 \mathrm{APs}$ at $20 \mathrm{~Hz}$ to mobilize the entire recycling pool. Unquenching the remaining vesicles with $\mathrm{Nh}_{4} \mathrm{Cl}$ uncovers the resting pool. (b) The same experiment as in (a) was performed, this time allowing for spontaneous release to occur after the recycling pool has been mobilized. A single neuron was stimulated with $900 \mathrm{APs}$ at $20 \mathrm{~Hz}$ to mobilize the entire recycling pool. Synapses were left for $15 \mathrm{~min}$ at room temperature in the presence of TTX to establish whether any vesicles could be released spontaneously. Finally, any remaining vesicles were unquenched by addition of $\mathrm{Nh}_{4} \mathrm{Cl}$. The size of the recycling pool and the resting pool of vesicles are shown for each cell. (c) Example images of the presynaptic terminals from the neuron shown in (b) after different treatments. Top from left to right: synapses at rest and after $900 \mathrm{APs}$ at $20 \mathrm{~Hz}$; bottom from left to right: synapses after spontaneous release for 15 min at room temperature, in TTX and $2 \mathrm{mM}$ calcium and after unquenching all vesicles with $\mathrm{Nh}_{4} \mathrm{Cl}$. Scale bar $=5 \mu \mathrm{m}$. Values are shown as mean \pm SEM. 
a

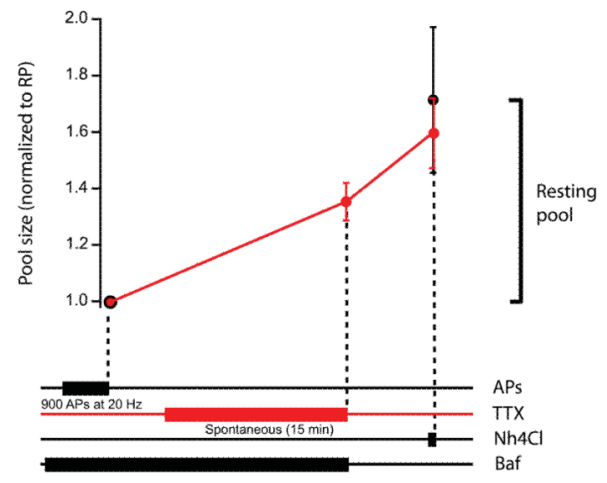

b

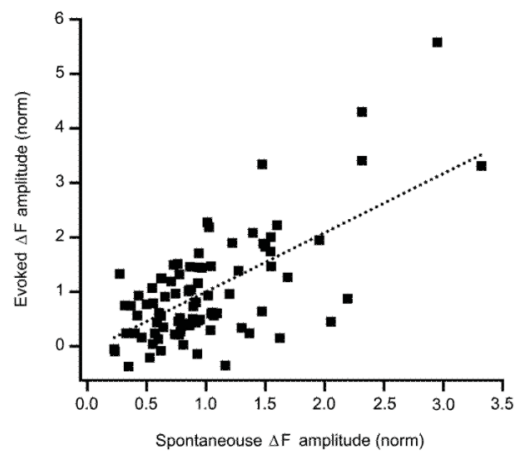

Figure 7. The spontaneous pool of vesicles corresponds to the resting pool

(a) The graph shows a plot of the change in fluorescence (normalized to the Recycling pool) following different treatments. After the entire recycling pool has been mobilized with a saturating stimulus of $900 \mathrm{APs}$ at $20 \mathrm{~Hz}$, neurons are either treated immediately with $\mathrm{Nh}_{4} \mathrm{Cl}$ (black trace, $\mathrm{n}=6$ cells) or allowed to release spontaneously for $15 \mathrm{~min}$ in TTX, at room temperature and subsequently treated with $\mathrm{Nh}_{4} \mathrm{Cl}$ (red trace, $\mathrm{n}=7$ cells). The diagram below gives a temporal representation of the experimental protocol. (b) Graph showing a plot of the change in fluorescence amplitude measured with sypHy for evoked release at individual synapses (black squares) as a function of the change in fluorescence for spontaneous release. The doted black line represents the best linear fit to the data (correlation coefficient $=0.62, p$ $<0.05)$. Values are shown as mean \pm SEM. 